\title{
INTERBLEND: Vinos obtenidos de la unión de vinos de dos o más países
}

\author{
Luis Rovello ${ }^{1, \text { a }}$ y Guillermo Pontis ${ }^{2}$ \\ ${ }^{1}$ ALACIMA - Full Agency, Director General, Martínez de Rosas 246, Mendoza, Argentina \\ ${ }^{2}$ UWA - Club del Vino, Director General, Via Zucchi 25, Monza, Italia
}

\begin{abstract}
Resumen. En el contexto de un mercado internacional cada vez más dinámico y competitivo, donde los consumidores toman cada vez más protagonismo en la cadena de valor, se presenta la iniciativa InterBlend que tiene como finalidad contribuir al aumento del consumo de la categoría vino, a través de una idea totalmente innovadora, despertando el interés en los nuevos consumidores para que se acerquen y vivan una nueva experiencia. InterBlend pretende trabajar en sintonía con las bodegas y los entes de regulación vitivinícola de los países miembros de la OIV, monitoreando, asistiendo y capacitando a sus miembros en esta nueva práctica de elaboración y comercialización de vinos de corte obtenidos de la unión de vinos de dos o más países. InterBlend se fundamenta en las opiniones de los consumidores de vino, tomando como eje de estudio sus gustos y preferencias, generados a partir de una base de encuestas y testimoniales a nivel mundial. InterBlend cree en un sector vitivinícola internacional competitivo en el cual se desarrollen negocios de manera responsable y sostenible.
\end{abstract}

\section{Quienes somos}

Interblend es una iniciativa que se presenta como un organismo de coordinación independiente que pretende trabajar en sintonía con las bodegas y los entes de regulación vitivinícola de los países miembros de la OIV (Organización Internacional de la Viña y el Vino); monitoreando, asistiendo y capacitando a sus miembros en la elaboración de vinos de corte obtenidos de la unión de vinos de dos o más países.

Nuestro compromiso es regular las prácticas de vinos de corte, bajo los lineamientos aprobados por los organismos de control de cada país y de la OIV.

Nuestro objetivo central pretende atender los gustos y pre ferencias de los nuevos consumidores, buscando elaborar vin os de calidad, sustentables y diversos.

\section{Objetivos}

- Fomentar la integración cultural y el consumo del vino a nivel mundial, resaltando cuidadosamente los atributos diferenciadores de cada terroir y región vitivinícola.

- Hacer foco en la tecnología, muy involucrados con la experiencia de cada consumidor.

- Fortalecer el vínculo entre lo privado y lo gubernamental.

- Incentivar a los países que no son miembros de la OIV a que se adhieran.

- Fortalecer la industria vitivinícola, participando en el análisis y elaboración de vinos de corte.

- Ser un punto de encuentro para los asociados, orientándolos en las diferentes fases del negocio sin afectar sus identidades propias.

a Autor de correspondencia: luisrovello@gmail.com; pontisguillermo@gmail.com

\section{3. Ámbito de participación y acción}

InterBlend representa los intereses de sus asociados ante diferentes organismos o instituciones del vino tanto en el orden nacional como internacional.

InterBlend cuenta con miembros observadores (organismos del vino de cada país) y miembros asociados (establecimientos vitivinícolas "bodegas"). Cada solicitud se estudia cuidadosamente para comprobar el cumplimiento de una serie de criterios de selección para la incorporación al programa InterBlend y el acceso a la membresía anual.

Mientras que el organismo InterBlend actúa como coordinador general, proporcionando asesoramiento y expandiendo el programa a nuevos países, los miembros (asociados y observadores) son los encargados de colaborar, implantar e informar sobre las actividades de este tipo de prácticas en sus respectivos países.

Se proyecta la auto sustentabilidad económica del Organismo, a través de la ejecución de dos modelos de negocios que en el largo plazo permitan la continuidad de los proyectos de interés para la industria. El primero consiste en el cobro de un royalty sobre las bodegas actuantes (de categoría miembro), mientras que el segundo incluye ingresos generados por el sello "InterBlend" y servicios asociados.

\section{Nuestra misión}

InterBlend tiene como objetivo promover un entorno social, económico y jurídico que favorezca el desarrollo sostenible y responsable de vinos de corte, obtenidos de la unión de vinos de dos o más países.

\section{Nuestras actividades y servicios son}

- Capacitación (Know How), promoción, representación y comunicación, en un diálogo constructivo,

This is an Open Access article distributed under the terms of the Creative Commons Attribution License 4.0, which permits unrestricted use, distribution, and reproduction in any medium, provided the original work is properly cited. 
responsable y permanente con las autoridades nacionales e internacionales pertinentes y otras partes interesadas con el fin de promover los intereses comunes de los miembros asociados (bodegas).

- La organización de reuniones y eventos InterBlend que aseguren una consulta adecuada con miras a la adopción y difusión de posiciones comunes.

- Información: recogida, análisis y difusión de información entre nuestros miembros con respecto a las áreas claves del programa InterBlend.

- La prestación de asesoramiento jurídico, técnico, económico y/o comercial relacionado con las actividades y buenas prácticas InterBlend.

- Desarrollo o coordinación de estudios o investigaciones Ad-hoc.

- Conformación de una plataforma única para debatir y adoptar posiciones e implementar acciones en el marco de una red profesional estructurada.

\section{Nuestra visión}

Ser un organismo clave que conduzca al diálogo, la coordinación y la promoción del sector vitivinícola internacional en todos los ámbitos políticos que afectan al negocio y al éxito de los vinos de corte, obtenidos de la unión de dos o más países.

\section{Nuestros valores}

\subsection{Excelencia}

InterBlend se ha comprometido a promover la excelencia de la identidad, la calidad y la imagen de los vinos de corte IB en todo el mundo.

\subsection{Responsabilidad}

Centrado en los intereses de los consumidores, InterBlend se compromete a promover pautas moderadas y responsables de consumo de vino IB.

\subsection{Sostenibilidad}

InterBlend se ha comprometido a promover prácticas de producción y comercio que sean ecológicas, equitativas y económicamente viables.

\subsection{Competitividad}

InterBlend se ha comprometido a contribuir a la mejora de la competitividad del sector vitivinícola en el mercado global, promoviendo:

- La elaboración y comercialización de vinos IB en un ambiente justo;

- La protección adecuada de los derechos de propiedad intelectual contra la usurpación, falsificación o cualquier otra forma de fraude;

- La investigación y la innovación.

\section{Metodología}

En el contexto de un mercado internacional cada vez más dinámico y competitivo, donde los consumidores toman cada vez más protagonismo en la cadena de valor, InterBlend se presenta como un organismo de coordinación independiente y de carácter privado que trabaja de la mano del máximo ente de Regulación Vitivinícola de cada país y en sintonía con los propósitos de la OIV, actuando como facilitador en las diferentes etapas de elaboración, producción y comercialización de todos los vinos elaborados a partir de un corte único con vinos de dos o más países.

InterBlend tiene como función principal la de fomentar el consumo de vino en las nuevas generaciones, estandarizando las normas de producción de esta nueva práctica a nivel internacional. Busca resaltar los atributos, historia y cultura de diferentes regiones vitivinícolas obteniendo así un "Blend de culturas". Su mayor responsabilidad es la de monitorear, asistir y capacitar a los diferentes actores involucrados en todo el proceso de control, para que se cumplan todas las reglamentaciones impuestas por los diferentes organismos fiscalizadores, atendiendo de cerca los intereses del nuevo consumidor.

Todos los estados miembros de la OIV están invitados a participar de esta nueva iniciativa, en la que los establecimientos vitivinícolas de cada país, a través de alianzas de trabajo con otros establecimientos vitivinícolas, podrán trabajar en conjunto para crear nuevos estilos de vino de corte, habiendo acordado previamente con InterBlend la metodología de trabajo, que estipula, por ejemplo, la cantidad de vino a elaborar, sede de fraccionamiento, selección y compra de insumos necesarios, lugar de estiba, logística, comercialización, etc.

InterBlend participa en las negociaciones internacionales para lograr acuerdos que faciliten el comercio exterior de los vinos de corte y su correcta inserción en el mercado. Será el nexo con el nuevo consumidor, instruyéndolo a través de una plataforma interactiva, la cual estará constantemente actualizada con información relevante del sector. Funcionará como punto de encuentro entre los consumidores y las bodegas involucradas (miembros asociados).

\section{Protección de las indicaciones geográficas y denominaciones de origen}

Las indicaciones geográficas (I.G.) y las denominaciones de origen (D.O.) protegen productos originarios del país o de una región o localidad, siempre que tengan una calidad, reputación u otra característica imputable a su origen geográfico. En el caso de las denominaciones de origen, deben presentar además factores naturales y humanos que inciden en la caracterización del producto.

InterBlend pretende trabajar en un ámbito de respeto científico-comercial, atendiendo las normativas vigentes, asociadas a las indicaciones geográficas y a las denominaciones de origen, contribuyendo en gran medida, a la comunicación leal del producto terminado.

InterBlend formulará recomendaciones y planteará a sus asociados la necesidad de demostrar una garantía de autenticidad de los productos, llevando el sello IB, en 
particular en cuanto a las menciones de etiquetado y de puesta en cada mercado.

\section{Impacto esperado}

- Renovar la imagen del vino y generar mayor confianza en los mercados.

- Reforzar el Top of Mind de la categoría vinos, sobre todo en los nuevos consumidores y público joven.

- Aumentar el consumo y las ventas en los mercados más exigentes.

- Favorecer la unión de las diferentes culturas.

- Incentivar a la implantación de viñedos.

- Afianzar las relaciones con comunidades y clima laboral entre países.

\section{Beneficio para los asociados}

\subsection{Bodegas Asociadas}

- Incorporación de un producto innovador a sus portfolios comerciales.

- Incremento en el Mix de ventas y facturado.

- Apertura de nuevos mercados.

- Oferta diferenciada para importadores y distribuidores.

- Posicionamiento para las bodegas miembro.

- Protagonismo y participación del enólogo.

- Soporte en materia de comunicación y distribución del mensaje.

\subsection{Organismos de Control / Fiscalización}

- Nuevos puestos de trabajo.

- Unión entre diferentes culturas.

- Mejora las relaciones con las comunidades.

- Definición de pautas para la producción y comercialización.

- Redefinición de procesos, con foco en el consumidor.

\subsection{Consumidores}

- Producto $100 \%$ innovador.

- Acerca nuevos consumidores al vino y fideliza a los existentes.

- Revolucionario.

- Mejora la experiencia de consumo y el vínculo con las marcas, productores y la gastronomía.

- Aprendizaje sobre diferentes culturas y características de cada fusión enológica, en relación a métodos de elaboración, terroirs, climas, etc.

\subsection{Comunidad Científica}

- Salto exponencial sobre el conocimiento científico en el área de alimentos y bebidas.

- Mejora las relaciones entre la comunidad científica y empresarial.

- Incremento acelerado de I+D.

- Factibilidad en la implementación de nuevos formatos.

\section{Eficiencia a través de la Innovación}

En este marco, abogamos por la creación y financiación de una plataforma de investigación vitivinícola para producir en forma estructurada y a largo plazo I+D, donde se pretende trabajar en:

- Definición de una hoja de ruta estratégica para los próximos 10 años;

- Creación de una red de excelencia que reúna a las comunidades científicas y profesionales;

- Fomentar la participación de las PYMES productoras de vino en los proyectos de investigación;

- Promover la difusión eficaz de los resultados de la investigación en el sector vitivinícola. 